\title{
Effects of the cholesteryl ester transfer protein inhibitor, TA-8995, on cholesterol efflux capacity and high-density lipoprotein particle subclasses
}

\author{
Julian C. van Capelleveen, MD, John J. P. Kastelein, MD, PhD*, \\ Aeilko H. Zwinderman, PhD, Sander J. H. van Deventer, MD, PhD, \\ Heidi L. Collins, PhD, Steven J. Adelman, PhD, Patrick Round, PhD, \\ John Ford, PhD, Daniel J. Rader, MD, PhD, G. Kees Hovingh, MD, PhD
}

Department of Vascular Medicine, Academic Medical Center, Amsterdam, The Netherlands (Drs van Capelleveen, Kastelein, and Hovingh); Dezima Pharma BV, Naarden, The Netherlands (Drs Kastelein, van Deventer, Round, and Ford); Department of Clinical Epidemiology and Biostatistics, Academic Medical Center, Amsterdam. The Netherlands (Dr Zwinderman); Department of Gastroenterology, Leiden University Medical Centre, Leiden, The Netherlands (Dr van Deventer); Vascular Strategies, Horsham, PA, USA (Drs Collins and Adelman); Xention Ltd, Cambridge, UK (Drs Round and Ford); and Department of Genetics, Perelman School of Medicine, University of Pennsylvania, PA, USA (Dr Rader)

\section{KEYWORDS:}

TA-8995;

CETP inhibitor; Cholesterol efflux capacity;

HDL;

Pre-Beta1 HDL
BACKGROUND: TA-8995 is a potent inhibitor of cholesteryl ester transfer protein (CETP) with beneficial effects on lipids and lipoproteins. The effect of TA-8995 on cholesterol efflux capacity (CEC), a measure of high-density lipoprotein (HDL) function, and HDL subparticle distribution is largely unknown.

OBJECTIVE: To assess the effect of the CETP inhibitor TA-8995 on ABCA1- and non-ABCA1driven CEC and on HDL particle distribution.

METHODS: Total, non-ABCA1-, and ABCA1-specific CEC from J774 cells and HDL subclass distribution assessed by two-dimensional gel electrophoresis were measured at baseline and after 12-week treatment in 187 mild-dyslipidemic patients randomized to placebo, $1 \mathrm{mg}, 5 \mathrm{mg}, 10 \mathrm{mg} \mathrm{TA}-8995$, or $10 \mathrm{mg}$ TA-8995 combined with $10 \mathrm{mg}$ rosuvastatin (NCT01970215).

RESULTS: Compared with placebo, total, non-ABCA1-, and ABCA1-specific CEC were increased dose dependently by up to $38 \%, 72 \%$, and $28 \%$, respectively, in patients randomized to $10 \mathrm{mg}$ of TA-8995. PreBeta-1 HDL, the primary acceptor for ABCA1-driven cholesterol efflux, was increased by $36 \%$. This increase in preBeta-1 HDL correlated significantly with the total and the ABCA1driven CEC increase, whereas the high-density lipoprotein cholesterol (HDL-C) increase did not.

CONCLUSION: TA-8995 dose dependently increased not only total and non-ABCA1-specific CEC but also ABCA1-specific CEC and preBeta-1 HDL particle levels. These findings suggest that TA-8995

* Corresponding author. Department of Vascular Medicine, Academic Medical Center, University of Amsterdam, Meibergdreef 9, 1105AZ Amsterdam, The Netherlands.

E-mail address: j.s.jansen@amc.uva.nl

Submitted March 24, 2016. Accepted for publication June 18, 2016. 
not only increases HDL-C levels but also promotes functional properties of HDL particles. This CETP inhibitor-driven preBeta-1 HDL increase is an important predictor of both ABCA1 and total CEC increase, independent of HDL-C increase. Whether these changes in HDL particle composition and functionality have a beneficial effect on cardiovascular outcome requires formal testing in a cardiovascular outcome trial.

(C) 2016 National Lipid Association. All rights reserved.

\section{Introduction}

Large prospective studies have shown that plasma levels of high-density lipoprotein cholesterol (HDL-C) levels are inversely related with cardiovascular disease (CVD). ${ }^{1}$ Findings derived from recent Mendelian randomization studies, however, showed that genetic variants with an effect on HDL-C levels carry no predictive value for CVD outcomes. ${ }^{2}$ In addition, therapeutic interventions that were designed to increase HDL-C failed to show an effect on CVD events, and these data have casted doubt on the causal relation between HDL-C and CVD outcomes. ${ }^{3-5}$

HDL has been shown to encompass several properties that protect against atherosclerosis, but the major antiatherogenic effect is believed to be its ability to remove excess cholesterol from macrophages in the arterial wall. ${ }^{6}$ Indeed, the capacity of plasma to mediate cholesterol efflux from macrophages is strongly predictive of future cardiovascular events, even after adjustment for plasma HDLC. ${ }^{7-9}$ This apparent discrepancy might be explained by nonfunctional HDL through oxidative modifications secondary to inflammation. ${ }^{10}$ Alternatively, the distribution of HDL particles may be skewed away from subfractions with a specific efflux receptor affinity. For example, ATPbinding cassette transporter A1 (ABCA1) transfers cellular free cholesterol to small nascent preBeta-1 HDL particles, whereas ABCG1 promotes the efflux of free cholesterol to larger, more mature alpha HDL particles. ${ }^{11,12}$

Cholesteryl ester transfer protein (CETP) transfers cholesteryl esters from HDL to apoB-containing lipoproteins, and CETP inhibitors were initially developed as HDL-C raising agents, in the assumption that raising HDL-C levels would result in CVD risk reduction. Indeed, CETP inhibitors were shown to increase HDL-C and apoA-1 levels, but the effect size of the different CETP inhibitors on these parameters varies greatly. ${ }^{3,4,13,14}$ To date, however, CVD outcome studies investigating the effect of HDL-C raising modalities on CVD events have failed to show a beneficial effect. ${ }^{3,4}$ These results have spurred a debate on the functionality of the HDL that is generated by CETP inhibitors, and it has been speculated that CETP inhibitors may promote large nonfunctional HDL particles. ${ }^{15}$ Against this background, it has become opportune to study the effects of CETP inhibition on the distribution of HDL subfractions and to measure the cholesterol efflux capacity (CEC) of plasma from patients treated with CETP inhibitors.
In a previous study, we found that total CEC was increased by TA-8995. ${ }^{16}$ In the present study, we explored the effects of TA-8995 treatment on non-ABCA1- and ABCA1-specific CEC, and we assessed CETP inhibitor driven changes on HDL subparticle distribution and their correlation with different cholesterol efflux capacities as well as with lipids and lipoproteins.

\section{Material and methods}

\section{Study design and participants}

The TULIP trial was a multicenter randomized, doublebind, placebo-controlled, parallel-group, phase 2 trial in 364 patients, distributed over 9 treatment arms. ${ }^{16}$ The study was conducted between August 2013 and July 2014 and was in compliance with the ethical principles in the Declaration of Helsinki, the International Conference on Harmonization/Good Clinical Practice and appropriate regulatory requirements. The protocol was reviewed and approved by the institutional review board of each participating center, and each patient provided written informed consent.

Details on study design have been reported. ${ }^{16}$ In short, patients aged 18 to 75 years with mild dyslipidemia were recruited, defined as low-density lipoprotein cholesterol (LDL-C) levels between 2.5 and $4.5 \mathrm{mmol} / \mathrm{L}$, HDL-C levels between 0.8 and $1.8 \mathrm{mmol} / \mathrm{L}$ and triglycerides below $4.5 \mathrm{mmol} / \mathrm{L}$. Major exclusion criteria were clinically manifest CVD, type 1 diabetes, poorly controlled type 2 diabetes (HBA1C over 8\%), or uncontrolled hypertension.

\section{Study procedures}

Plasma samples used for the analysis were obtained at baseline and week 12, in a fasted state. CEC was measured by Vascular Strategies (Horsham, PA) according to methods previously described ${ }^{7}$ in subjects randomized to placebo $(\mathrm{n}=37), 1 \mathrm{mg}$ of TA-8995 ( $=37), 5 \mathrm{mg}$ of TA-8995 $(\mathrm{n}=39), 10 \mathrm{mg}$ of TA-8995 $(\mathrm{n}=35)$ as monotherapy, and $10 \mathrm{mg}$ of TA-8995 combined with $10 \mathrm{mg}$ rosuvastatin $(\mathrm{n}=39)$. In short, J774 cells were radiolabeled with $2 \mathrm{uCi}$ of ${ }^{3} \mathrm{H}$-cholesterol per milliliter. ABCA1 was upregulated by 6-hour incubation with $0.3 \mathrm{mM}$ of 8-(4-chlorophenylthio) -cyclic-AMP. Medium, containing $2.8 \%$ apolipoprotein 
B-depleted plasma, was then added for 4 hours to cAMP stimulated and unstimulated cells. Liquid scintillation counting was used to quantify the effluxed ${ }^{3} \mathrm{H}$-cholesterol to the medium. Total CEC is defined as the efflux measured from cAMP-stimulated J774 cells. Non-ABCA1-specific CEC was measured from unstimulated J774 cells. ABCA1specific CEC was calculated as the difference between cAMP-stimulated (total) and unstimulated (non-ABCA1) cells. In the initial report, we presented the data for total CEC. ${ }^{16}$ After analysis of the preBeta- 1 HDL twodimensional (2D)-gelelectrophoresis data (see in the following section), we additionally measured ABCA1- and nonABCA1-specific CEC (cAMP stimulated and unstimulated) in plasma samples that were frozen and stored at $-80^{\circ} \mathrm{C}$.

HDL subparticle distribution (preBeta-1, preBeta-2, and alpha 1-4) was measured in the placebo, $5 \mathrm{mg}$ of TA-8995, $10 \mathrm{mg}$ of TA-8995, and $10 \mathrm{mg}$ of TA-8995 combined with $10 \mathrm{mg}$ of rosuvastatin treatment arms using 2D gel electrophoresis in plasma from placebo, $5 \mathrm{mg}$ of TA$8995,10 \mathrm{mg}$ of TA-8995, and $10 \mathrm{mg}$ of TA-8995 plus 10-mg rosuvastatin-treated subjects according to methods previously published. ${ }^{17}$

\section{Statistical analysis}

Differences in CEC (total, non-ABCA1-, and ABCA1specific) and HDL subparticle distribution were analyzed as percent change from baseline using analysis of covariance with baseline values for the respective parameters as a covariate. HDL subparticle distribution by 2D gel electrophoresis is reported for the combined 5-mg TA-8995 and 10-mg TA-8995 monotherapy treatment arms. To address the contribution of preBeta-1 HDL to cholesterol efflux, multiple linear regression analysis was used to report the proportion of variance explained for specific models. Models with and without percent change from baseline for HDL-C and preBeta-1 HDL were compared in terms of $\mathrm{R}^{2}$ change. Regression analysis was performed for base covariates (model 1) and either addition of percent change in HDL-C from baseline (model 2) or addition of percent change in preBeta-1 HDL from baseline (model 3). Finally, percent change in preBeta-1 HDL was added to model 2, resulting in model 4 . This model represents the proportion of variance in CEC additionally explained by preBeta-1 HDL independent of HDL-C increase.

\section{Results}

\section{Baseline characteristics}

The overall baseline characteristics and predefined end points of the phase 2 study were previously reported. ${ }^{16} \mathrm{We}$ measured CEC and HDL particle subclasses in a total of 187 participants randomized to receive treatment with placebo $(\mathrm{n}=37), 1 \mathrm{mg}$ of TA-8995 $(\mathrm{n}=37), 5 \mathrm{mg}$ of
TA-8995 ( $\mathrm{n}=39), 10 \mathrm{mg}$ of TA-8995 ( $\mathrm{n}=35)$, or $10 \mathrm{mg}$ of TA-8995 combined with $10 \mathrm{mg}$ of rosuvastatin $(\mathrm{n}=39)$. Baseline characteristics were similar between treatment groups (Supplemental Table S1).

\section{Cholesterol efflux capacity}

Total CEC increased in patients randomized to TA-8995, and this effect was found to be dose dependent by $17 \%$, $33 \%$, and $38 \%$ in the patients receiving $1 \mathrm{mg}, 5 \mathrm{mg}$ and $10 \mathrm{mg}$ TA-8995, respectively, while CEC increased $31 \%$ in patients on 10-mg TA-8995 plus 10-mg rosuvastatin combination therapy (all $P<.001$ compared with placebo, Fig. 1A), as was recently reported. ${ }^{16}$ Non-ABCA1specific CEC increased by $38 \%, 62 \%$, and $72 \%$ in the groups receiving $1 \mathrm{mg}, 5 \mathrm{mg}$, and $10 \mathrm{mg}$ of TA-8995, respectively, whereas a $67 \%$ increase was observed in patients randomized to $10 \mathrm{mg}$ of TA-8995 in combination with $10 \mathrm{mg}$ of rosuvastatin (all $P<.001$ compared with placebo, Fig. 1B). ABCA1-specific CEC was increased by $14 \%, 25 \%$, and $28 \%$ for 1,5 , and $10 \mathrm{mg}$ TA- 8995 monotherapy $(P<.05$ for $5 \mathrm{mg}$ and $P<.01$ for $10 \mathrm{mg}$ TA-8995 compared with placebo). Combination therapy of 10-mg TA-8995 plus 10-mg rosuvastatin resulted in a nonsignificant $16 \%$ increase in ABCA1-specific CEC, when compared with placebo. The difference between 10-mg TA-8995 monotherapy and 10-mg TA-8995 in combination with 10-mg rosuvastatin was nonsignificant for all analyses.

\section{HDL subparticle distribution}

The effect of TA-8995 treatment on HDL subparticle distribution was measured by $2 \mathrm{D}$-gel-electrophoresis in subjects receiving placebo, $5 \mathrm{mg}$ of TA-8995, $10 \mathrm{mg}$ of TA8995 , and $10 \mathrm{mg}$ of TA-8995 combined with $10 \mathrm{mg}$ of rosuvastatin therapy, Figure 2. The change from baseline was similar for 5-mg and 10-mg TA-8995 monotherapy; therefore, we report the combined results for these groups (results for separate groups; Supplemental Tables S2 and S3).

As expected, large alpha-1 HDL particle levels increased by $350 \%$ and $352 \%(P<.001)$ in patients receiving monotherapy and combination therapy, respectively. Alpha-2 and alpha-4 particles were not significantly changed in any treatment group, whereas alpha-3 HDL was significantly decreased by $17 \%$ from baseline in both monotherapy and combination therapy $(P<.001)$.

Finally, preBeta-1 HDL, a small discoidal lipid-poor particle that is the primary acceptor for ABCA1-driven cholesterol efflux, was significantly increased by $36 \%$ for TA-8995 monotherapy $(P<.001)$; on $10-\mathrm{mg}$ TA-8995 combined with $10-\mathrm{mg}$ rosuvastatin, there was a trend toward an increase (22\%), but this was not statistically significant. The larger preBeta- 2 HDL increased by $66 \%$ in patients on monotherapy and by $64 \%$ in patients receiving combination therapy with 10 -mg rosuvastatin $(P<.001)$. 
A

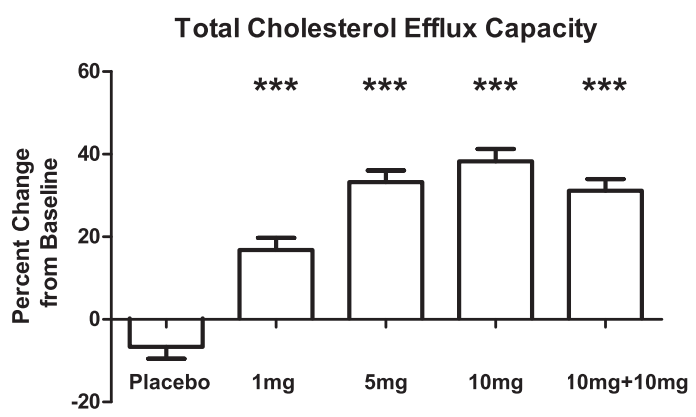

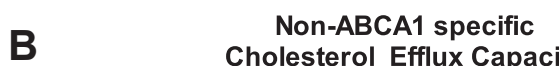

Cholesterol Efflux Capacity

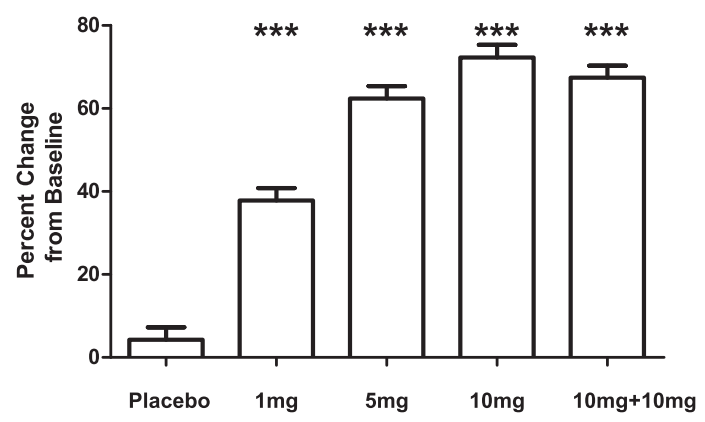

C

ABCA1-specific Cholesterol Efflux Capacity

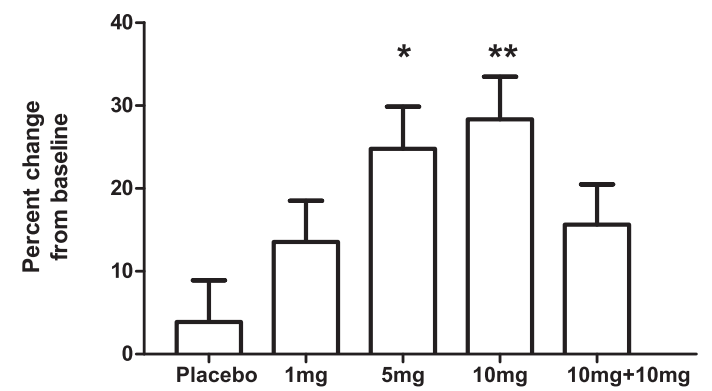

Figure 1 (A-C) Total (A), non-ABCA1 (B), and ABCA1-specific (C) cholesterol efflux capacity as percent change from baseline. Data are presented as least squares means \pm standard error of the mean. $* P<.05$, $* * P<.01, * * * P<.001$ compared with placebo. Placebo ( $\mathrm{n}=37)$, " $1 \mathrm{mg}$ " is treatment with $1 \mathrm{mg}$ of TA-8995 monotherapy (n = 37), " $5 \mathrm{mg}$ " is treatment with $5 \mathrm{mg}$ of TA-8995 monotherapy ( $\mathrm{n}=39)$, " $10 \mathrm{mg}$ " is treatment with $10 \mathrm{mg}$ of TA-8995 monotherapy $(\mathrm{n}=35)$, " $10 \mathrm{mg}+10 \mathrm{mg}$ " is combination therapy of $10 \mathrm{mg}$ of TA-8995 plus $10 \mathrm{mg}$ rosuvastatin $(\mathrm{n}=39)$.

\section{Correlation analysis}

The correlation of HDL-C and preBeta-1 HDL increases to $\mathrm{CEC}$ increases as coefficient of determination $\left(\mathrm{R}^{2}\right)$ was assessed using multiple linear regression analysis. The results of these analyses are summarized in Table 1.

HDL-C increase at week 12 was not significantly correlated with total and $\mathrm{ABCA1}$-specific $\mathrm{CEC}$ increase, whereas it was significantly correlated with non-ABCA1specific CEC increase $\left(\mathrm{R}^{2}\right.$ change $\left.0.08, P<.001\right)$. In contrast, preBeta-1 HDL increase was significantly correlated with total and ABCA1-specific CEC increase $\left(\mathrm{R}^{2}\right.$ change 0.16 and 0.15 , respectively, $P<.001$ ). The correlation with non-ABCA1-specific CEC was smaller $\left(\mathrm{R}^{2}\right.$ change $0.04, P<.05$ ). Correction for HDL-C increase did not significantly change the correlation of preBeta-1 HDL with CEC (model 4).

Baseline total CEC was explained for a substantial proportion by HDL-C and preBeta-1 HDL levels. The contribution of preBeta-1 HDL to ABCA1-specific CEC at baseline was larger compared with HDL-C, and contribution of preBeta-1 to non-ABCA1-specific cholesterol efflux was small. Altogether, these findings indicate that the increase in preBeta-1 HDL explains not only a large proportion of variance in ABCA1-specific CEC but also that of total CEC, independent of HDL-C increase, further underlining the importance of preBeta-1 HDL increase in relation to the observed increase of CEC.

\section{Discussion}

Treatment with the CETP inhibitor TA-8995 led to a significant increase of total, non-ABCA1-, and ABCA1specific $\mathrm{CEC}$ as well as a significant increase in preBeta-1 HDL levels. The increase in HDL-C did not correlate with total or ABCA1-specific CEC increase, whereas the increase in preBeta-1 HDL had a significant correlation with total and ABCA1-specific CEC. These findings not only underline the importance of the preBeta-1 HDL increase in relation to total CEC, a predictor of incident CVD events, but also confirm the earlier notion that HDL-C levels and HDL functionality as assessed by CEC are not necessarily interchangeable. Our data indicate that CETP inhibition by means of TA- 8995 increases preBeta-1 HDL levels and HDL particle functionality beyond a mere increase in HDL-C levels.

Although low HDL-C levels are among the strongest epidemiological risk factors for CVD, both genetic studies 
A

\section{HDL subparticle distribution}

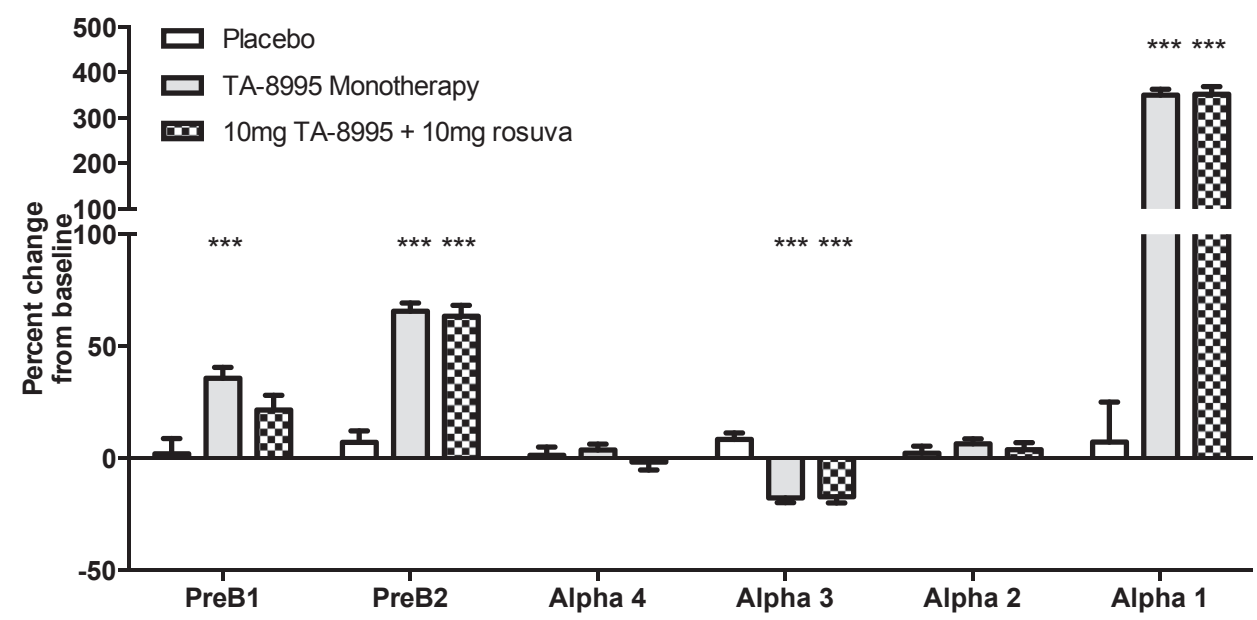

B

PreBeta1 HDL

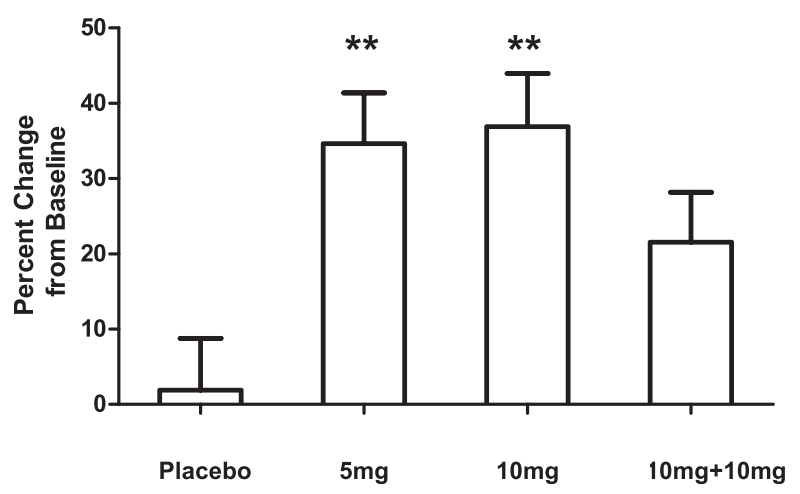

Figure 2 High-density lipoprotein (HDL) subparticle distribution as percent change from baseline as measured by $2 \mathrm{D}$ gel electrophoresis. Data are presented as least squares means \pm standard error of the mean. (A) shows preBeta-1, preBeta-2, alpha-4, alpha-3, alpha-2, and alpha-1 results for placebo $(\mathrm{n}=36)$, monotherapy $(5 \mathrm{mg}+10 \mathrm{mg}$ TA-8995, $\mathrm{n}=72)$ and combination therapy: $10 \mathrm{mg}$ of TA-8995 plus $10 \mathrm{mg}$ rosuvastatin $(\mathrm{n}=39)$. (B) shows the specific preBeta-1 data for placebo $(\mathrm{n}=36)$, "5 mg" is treatment with $5 \mathrm{mg}$ of TA-8995 $(\mathrm{n}=36)$, "10 mg" is treatment with $10 \mathrm{mg}$ of TA-8995 ( $=34)$ and " $10 \mathrm{mg}+10 \mathrm{mg}$ " is combination therapy $10 \mathrm{mg}$ TA-8995 plus $10 \mathrm{mg}$ rosuvastatin $(\mathrm{n}=39)$. $* P<.05, * * P<.01, * * * P<.001$ compared with placebo.

and the outcome of clinical trials aimed to increase HDL-C levels have casted doubt on the clinical utility of increasing total HDL-C levels. These findings have shifted the HDL-C hypothesis toward HDL functionality as a mediator of HDLrelated atheroprotection. ${ }^{2}$ One of the key antiatherogenic properties of the HDL particle is its role in the process of efflux of cholesterol from peripheral tissues to the circulation, where the HDL particle is the main acceptor. In recent years, in vitro models have been developed to reliably and reproducibly measure this capacity. ${ }^{7} \mathrm{CEC}$, measured by the same methodology as in our study, was previously shown to be strongly and inversely related to coronary heart disease in a cross-sectional approach and in large prospective cohorts. ${ }^{7-9}$ It is of note that HDL-C levels were only a weak predictor for cholesterol efflux in those studies.

Although all CETP inhibitors have been shown to induce an increase in HDL-C levels, the effects of dalcetrapib vary to a great extent from those observed for the potent CETP inhibitors anacetrapib, evacetrapib, and TA-8995. HDL-C levels were shown to increase by $33 \%$ in patients randomized to a daily dose of $300 \mathrm{mg}$ of dalcetrapib, whereas anacetrapib, evacetrapib, and TA8995 achieved HDL-C increases up to $179 \%$. $^{4,13,14,16}$ Similarly, the effects of CETP inhibitors on HDL-functionality and CEC vary greatly. A clinical trial investigating the CETP inhibitor dalcetrapib in patients with acute coronary syndrome showed that dalcetrapib increased total CEC by 9.5\% with no effect on ABCA1-specific CEC or preBeta-1 HDL levels. ${ }^{18}$ In contrast, treatment with the CETP inhibitors anacetrapib and evacetrapib has been shown to increase both total CEC and ABCA1-specific CEC, to about the same extent as TA-8995. ${ }^{19,20}$ Hence, the increase of CEC is likely a CETP inhibitor class effect, with the potent compounds, anacetrapib, evacetrapib, and TA-8995, having a more pronounced effect on total and ABCA1-driven components of CEC. 
Table 1 Contribution of high-density lipoprotein cholesterol (HDL-C) and PreBeta1 high-density lipoprotein (HDL) to cholesterol efflux capacity (CEC) levels at baseline and after 12 weeks treatment with TA $=8995$ as regression coefficient of determination

\begin{tabular}{|c|c|c|c|c|c|c|c|c|c|c|}
\hline \multirow[b]{2}{*}{ Model } & \multirow{2}{*}{$\frac{\text { Baseline values }}{\text { Covariates }}$} & \multicolumn{3}{|c|}{ Total CEC } & \multicolumn{3}{|c|}{ ABCA1-specific CEC } & \multicolumn{3}{|c|}{ Non-ABCA1-specific CEC } \\
\hline & & $\overline{R^{2}}$ & $\mathrm{R}^{2}$ change & $P$ value & $\overline{R^{2}}$ & $\mathrm{R}^{2}$ change & $P$ value & $\overline{\mathrm{R}^{2}}$ & $\mathrm{R}^{2}$ change & $P$ value \\
\hline Model 1 & Age, gender, BMI, smoking status & 0.03 & - & - & 0.02 & - & - & 0.09 & - & - \\
\hline Model 2 & Model $1+$ base HDL-C & 0.17 & 0.14 & $<.001$ & 0.04 & 0.02 & ns & 0.39 & 0.31 & $<.001$ \\
\hline Model 3 & Model $1+$ base prebeta1 HDL & 0.25 & 0.22 & $<.001$ & 0.12 & 0.10 & $<.001$ & 0.15 & 0.06 & $<.01$ \\
\hline Model 4 & $\begin{array}{l}\text { Model } 2+\text { base PreBeta1 HDL } \\
12 \text { wk TA-8995 monotherapy }\end{array}$ & 0.31 & 0.14 & $<.001$ & 0.12 & 0.08 & $<.001$ & 0.40 & 0.01 & ns \\
\hline Model 1 & $\begin{array}{l}\text { Age, gender, BMI, smoking status, } \\
\text { baseline cholesterol efflux } \\
\text { parameter }\end{array}$ & 0.28 & - & - & 0.54 & - & - & 0.56 & - & - \\
\hline Model 2 & $\begin{array}{l}\text { Model } 1+\% \text { HDL-C increase } \\
\text { from baseline }\end{array}$ & 0.29 & 0.02 & ns & 0.56 & 0.02 & ns & 0.64 & 0.08 & $<.001$ \\
\hline Model 3 & $\begin{array}{l}\text { Model } 1+\% \text { preBeta } 1 \text { increase } \\
\text { from baseline }\end{array}$ & 0.43 & 0.16 & $<.001$ & 0.69 & 0.15 & $<.001$ & 0.59 & 0.04 & $<.05$ \\
\hline Model 4 & $\begin{array}{l}\text { Model } 2+\% \text { PreBeta } 1 \text { increase } \\
\text { from baseline }\end{array}$ & 0.44 & 0.14 & $<.001$ & 0.69 & 0.14 & $<.001$ & 0.66 & 0.02 & $<.05$ \\
\hline
\end{tabular}

BMI, body mass index; HDL, high-density lipoprotein; HDL-C, high-density lipoprotein cholesterol; ns, nonsignificant.

Multiple linear regression analysis with total, ABCA1- and non-ABCA1-specific CEC levels at baseline and after 12 weeks of treatment with TA-8995 monotherapy as independent parameters and specified models $1-4$ as predictors. Results are presented as $R^{2}, R^{2}$ change, and corresponding $P$ value for each specified model.

Among others, CETP inhibition leads to the formation of larger, more mature HDL particles, which is reflected in the robust increase in large alpha-1 HDL particles up to $350 \%$ observed in our study. As non-ABCA1-specific efflux is mainly driven by ABCG1 and SRB1 efflux to large HDL particles ${ }^{11,12}$ this fits with the observed increase in nonABCA1-specific CEC up to $72 \%$ at the $10 \mathrm{mg}$ dose of TA8995. However, the finding that ABCA1-specific CEC and small lipid-poor preBeta-1 HDL levels, the primary acceptor for ABCA1-mediated efflux, are increased on CETP inhibition is counterintuitive and poorly understood. To underline the importance of preBeta-1 HDL increase in relation to CEC, we showed that preBeta-1 HDL, independent of HDL-C increase, is an important predictor not only of ABCA1-specific but also of total CEC increase. In contrast, HDL-C increase was not correlated with total CEC increase, despite the $179 \%$ increase in HDL-C from baseline.

Currently, it is largely unknown how preBeta-1 HDL is synthesized, and the mechanism linking CETP inhibition with increased preBeta-1 levels remains to be elucidated. One of the possible explanations is that CETP inhibition results in increased apoA-1 synthesis from the liver, which consequently leads to increased plasma preBeta-1 HDL levels. It has been shown that a significant fraction of apoA1 undergoes intracellular lipidation and is released as nascent HDL from hepatocytes. ${ }^{21}$ Alternatively, preBeta-1 HDL may originate from the degradation of very low-density lipoprotein and other triglyceride-rich lipoproteins, which are a potential source of preBeta-1 HDL. ${ }^{22}$ Indeed, it was recently shown that CETP inhibition does result in increased clearance and lipolysis of triglyceride-rich lipoproteins. ${ }^{23}$
It is anticipated that patients who would potentially qualify for CETP inhibition are likely to be cotreated with statins. Therefore, it is reassuring that total and nonABCA1-specific CEC were still significantly increased in patients randomized to the combination of 10-mg TA-8995 and 10-mg rosuvastatin. However, it was clearly diminished in patients on dual therapy, compared with patients receiving TA-8995 alone. In fact, it was recently shown that statin treatment actually lowers both preBeta-1 levels and ABCA1-specific and total CEC. ${ }^{20}$ Hence, the observed nonsignificant increase in ABCA1-specific CEC in patients randomized to TA-8995 combined with a statin is likely caused by the reduction of CEC by statin treatment.

Recently, the ACCELERATE trial, investigating the effect of evacetrapib in patients with established CVD at high residual risk, was terminated for futility, ${ }^{24}$ fueling the question why the efflux increase has not resulted in clinical benefit. No formal answer can be provided because study results and a complete data analysis are still to be awaited. Nevertheless, baseline characteristics and sample size considerations have been recently reported and provide some clues for the lack of clinical benefit. First, baseline LDL-C of $2.10 \mathrm{mmol} / \mathrm{L}$ was low and indicative of a wellcontrolled population, which was also reflected by $98 \%$ of study population receiving statin therapy. ${ }^{25}$ Absolute LDL$\mathrm{C}$ reduction will be small in this population. A second concern is the diminished efficacy of evacetrapib to reduce apoB levels (as a substitute for low-density lipoprotein [LDL] particle numbers) in combination with statins compared with evacetrapib monotherapy, ${ }^{14}$ an effect also observed for TA-8995 in the TULIP trial. ${ }^{16}$ LDL-C lowering 
by CETP inhibition combined with statins is partly a reflection of CETP-mediated intravascular remodeling of LDL particles and only partly the consequence of LDL receptor mediated clearance of LDL particles. Therefore, the LDL$\mathrm{C}$ reduction by CETP inhibition in combination with statins will likely overestimate the potential CVD risk reduction. Third, ACCELERATE-enrolled high-risk coronary artery disease patients of which an unexpected large proportion were diabetic patients $(63.7 \%)$, and efflux potential never investigated in either category. ${ }^{20}$ Fourth, over $30 \%$ of participants in ACCELERATE experienced a recent acute coronary syndrome, on average 5.5 months before randomization. Previous LDL-C intervention studies have taught us to be cautious in our expectation of clinical benefit from LDL-C lowering in the first year after acute coronary syndrome, as a proportion of subsequent events are considered nonmodifiable or refractory to lipid-lowering interventions. In combination with the relatively short follow-up time of 25 months, all these factors combined might have seriously restricted power, considering the small sample size of 12.000 patients. In contrast to ACCELERATE, the phase III study REVEAL, investigating the potent CETP inhibitor anacetrapib has recently passed futility assessment. ${ }^{26}$ Where study power is an issue in ACCELERATE, REVEAL has enrolled over 30,000 subjects and is therefore assumed to have superior power over ACCELERATE.

Finally, it should be stressed that ACCELERATE was never designed or powered to test the clinical utility of raising CEC, and therefore, this important clinical question remains to be answered. Post-hoc analyses from ACCELERATE and REVEAL will have to be awaited.

In conclusion, we report that potent CETP inhibition by TA-8995 raises not only non-ABCA1-specific CEC but also ABCA1-specific efflux and small nascent preBeta-1 HDL levels. The preBeta-1 HDL increase is an important predictor of both ABCA1-and total CEC increase, independent of HDL-C increase. The mechanism linking CETP inhibition and preBeta-1 HDL formation as well as the role of statin therapy in this process remain to be determined. CETP inhibition with TA-8995 has now been linked to large decreases of atherogenic lipoproteins, as well as to an improvement in the functionality of antiatherogenic lipoproteins, a similar profile as shown for evacetrapib and anacetrapib before. The recent termination for futility of ACCELERATE might well be due to specific trial-related issues in combination with untoward consequences of the combination of statin and CETP inhibitors. We will further need to wait for the final results of REVEAL, which recently did pass futility assessment, for a final verdict on CEC induction by CETP inhibition as a therapeutic target.

\section{Acknowledgment}

Authors' contributions: All authors have substantially contributed to the article and approved the final article. P.R., J.F., G.K.H., S.J.H.v.D., and J.J.P.K. were responsible for the conception and design of the study; P.R., J.F., H.L.C., S.J.A., A.H.Z., and J.C.v.C. were responsible for analysis and interpretation of the data. The article was drafted by J.C.v.C., G.K.H., and J.J.P.K. and was critically revised by D.J.R. and S.J.H.v.D.

J.C.v.C. reports none; J.J.P.K. reports personal fees from Dezima Pharmaceuticals during the conduct of the study; personal fees from Cerenis, The Medicines Company, CSL Behring, Amgen, Sanofi, Regeneron, Eli Lilly, Genzyme, Aegerion, Esperion, AstraZeneca, Omthera, Pronova, Vascular Biogenics, Boehringer Ingelheim, Catabasis, AtheroNova, UniQure, Novartis, Merck, Ionis Pharmaceuticals, and Kowa; A.H.Z. reports none; S.J.H.v.D. is a managing partner at Forbion Capital Partners, which owns equity in Dezima Pharma; HLC and SJA are employees of Vascular Strategies; P.R. and J.F. are employees of Xention; D.J.R. serves on the advisory boards of Aegerion, Alnylam, Eli Lilly, Merck, Novartis, and Sanofi; G.K.H. reports that his institution has received funding from Dezima, Amgen, Pfizer, Sanofi, Regeneron, AstraZeneca, Genzyme, Cerenis, Synageva, Roche, ISIS Pharmaceuticals, Kowa, and Merck for undertaking clinical trials related to various forms of lipid-lowering medication and consulting fees from Amgen, Pfizer, Roche, Regeneron, and Sanofi.

\section{Financial disclosure}

This work was supported by Dezima Pharma. G.K.H. is holder of a Vidi grant (016.156.445) from the Netherlands Organisation for Scientific Research (NWO) and is supported by a grant from the CardioVascular Research Initiative (CVON2011-19; Genius) and the European Union (TransCard: FP7-603091-2). J.J.P.K. is a recipient of the Lifetime Achievement Award of the Netherland Heart Foundation (NHS, project number 2010T082).

\section{References}

1. Di Angelantonio E, Sarwar N, Perry P, et al. Major lipids, apolipoproteins, and risk of vascular disease. JAMA. 2009;302(18):1993-2000.

2. Rader DJ, Hovingh GK. HDL and cardiovascular disease. Lancet. 2014;384(9943):618-625.

3. Barter PJ, Caulfield M, Eriksson M, et al. Effects of torcetrapib in patients at high risk for coronary events. $N$ Engl J Med. 2007;357(21): 2109-2122.

4. Schwartz GG, Olsson AG, Abt M, et al. Effects of dalcetrapib in patients with a recent acute coronary syndrome. N Engl J Med. 2012; 367(22):2089-2099.

5. Parish S, Tomson J, Wallendszus K, et al. Effects of extended-release niacin with laropiprant in high-risk patients. N Engl J Med. 2014; 371(3):203-212.

6. Rosenson RS, Brewer HB, Davidson WS, et al. Cholesterol efflux and atheroprotection: advancing the concept of reverse cholesterol transport. Circulation. 2012;125(15):1905-1919.

7. Khera AV, Cuchel M, de la Llera-Moya M, et al. Cholesterol efflux capacity, high-density lipoprotein function, and atherosclerosis. $N$ Engl J Med. 2011;364(2):127-135. 
8. Rohatgi A, Khera A, Berry JD, et al. HDL cholesterol efflux capacity and incident cardiovascular events. N Engl J Med. 2014;371(25): 2383-2393.

9. Saleheen D, Scott R, Javad S, et al. Association of HDL cholesterol efflux capacity with incident coronary heart disease events: a prospective case-control study. Lancet Diabetes Endocrinol. 2015;3(7): 507-513.

10. Rosenson RS, Brewer HB, Ansell BJ, et al. Dysfunctional HDL and atherosclerotic cardiovascular disease. Nat Rev Cardiol. 2016;13(1): 48-60.

11. de la Llera-Moya M, Drazul-Schrader D, Asztalos BF, Cuchel M, Rader DJ, Rothblat GH. The ability to promote efflux via ABCA1 determines the capacity of serum specimens with similar highdensity lipoprotein cholesterol to remove cholesterol from macrophages. Arterioscler Thromb Vasc Biol. 2010;30(4):796-801.

12. Rosenson RS, Brewer HB, Chapman MJ, et al. HDL measures, particle heterogeneity, proposed nomenclature, and relation to atherosclerotic cardiovascular events. Clin Chem. 2011;57(3): 392-410.

13. Cannon CP, Shah S, Dansky HM, et al. Safety of anacetrapib in patients with or at high risk for coronary heart disease. $N$ Engl J Med. 2010;363(25):2406-2415.

14. Nicholls SJ, Brewer HB, Kastelein JJP, et al. Effects of the CETP inhibitor evacetrapib administered as monotherapy or in combination with statins on HDL and LDL cholesterol: a randomized controlled trial. JAMA. 2011;306(19):2099-2109.

15. Brewer HB. Increasing HDL cholesterol levels. N Engl J Med. 2004; 350(15):1491-1494.

16. Hovingh GK, Kastelein JJ, van Deventer SJ, et al. Cholesterol ester transfer protein inhibition by TA-8995 in patients with mild dyslipidaemia (TULIP): a randomised, double-blind, placebo-controlled phase 2 trial. Lancet. 2015;386:452-460.

17. Asztalos BF, Roheim PS, Milani RL, et al. Distribution of ApoA-Icontaining HDL subpopulations in patients with coronary heart disease. Arterioscler Thromb Vasc Biol. 2000;20(12):2670-2676.
18. Ray KK, Ditmarsch M, Kallend D, et al. The effect of cholesteryl ester transfer protein inhibition on lipids, lipoproteins, and markers of HDL function after an acute coronary syndrome: the dal-ACUTE randomized trial. Eur Heart J. 2014;35(27):1792-1800.

19. Yvan-Charvet L, Kling J, Pagler T, et al. Cholesterol efflux potential and antiinflammatory properties of high-density lipoprotein after treatment with niacin or anacetrapib. Arterioscler Thromb Vasc Biol. 2010; 30(7):1430-1438.

20. Nicholls SJ, Ruotolo G, Brewer HB, et al. Cholesterol efflux capacity and pre-beta-1 HDL concentrations are increased in dyslipidemic patients treated with evacetrapib. J Am Coll Cardiol. 2015;66(20): 2201-2210.

21. Chisholm JW, Burleson ER, Shelness GS, Parks JS. ApoA-I secretion from HepG2 cells: evidence for the secretion of both lipid-poor apoA-I and intracellularly assembled nascent HDL. J Lipid Res. 2002;43(1): 36-44.

22. Miyazaki O, Fukamachi I, Mori A, et al. Formation of preB1-HDL during lipolysis of triglyceride-rich lipoprotein. Biochem Biophys Res Commun. 2009;379(1):55-59.

23. Millar JS, Reyes-Soffer G, Jumes P, et al. Anacetrapib lowers LDL by increasing ApoB clearance in mildly hypercholesterolemic subjects. $J$ Clin Invest. 2015;125(6):2510-2522.

24. Eli-Lilly. Lilly to discontinue development of evacetrapib for high-risk atherosclerotic cardiovascular disease. 2015 Available at: http://lilly. mediaroom.com/index.php?s $=9042 \&$ item $=137476$. Accessed July 18,2016

25. Nicholls SJ. Impact of the cholesteryl ester transfer protein inhibitor evacetrapib on cardiovascular outcome. In: Annual Scientific Sessions of the American College of Cardiology (ACC). 2016 Available at: http:// clinicaltrialresults.org/Slides/ACC\%202016/Nicholls_ACCELERATE. pdf. Accessed July 18, 2016.

26. Merck. Merck provides update on REVEAL Outcomes Study. 2015 Available at: http://www.mercknewsroom.com/news-release/research -and-development-news/merck-provides-update-reveal-outcomes-study. Accessed July 18, 2016. 
Table S1 Baseline characteristics per treatment group

\begin{tabular}{|c|c|c|c|c|c|}
\hline Characteristics & Placebo $(n=37)$ & $\begin{array}{l}1 \mathrm{mg} \text { TA-8995 } \\
(\mathrm{n}=37)\end{array}$ & $\begin{array}{l}5 \mathrm{mg} \text { TA-8995 } \\
(\mathrm{n}=39)\end{array}$ & $\begin{array}{l}10 \mathrm{mg} \text { TA }-8995 \\
(n=35)\end{array}$ & $\begin{array}{l}10 \mathrm{mg} \text { TA- } 8995+10 \mathrm{mg} \\
\text { rosuvastatin }(\mathrm{n}=39)\end{array}$ \\
\hline Age (y) & $64.9 \pm 5.7$ & $66.2 \pm 6.1$ & $65.2 \pm 7.7$ & $65.9 \pm 4.8$ & $63.4 \pm 6.1$ \\
\hline BMI $\left(\mathrm{kg} / \mathrm{m}^{2}\right)$ & $26.1 \pm 1.8$ & $26.5 \pm 2.8$ & $26.3 \pm 2.7$ & $25.8 \pm 2.5$ & $26.3 \pm 3.5$ \\
\hline Total cholesterol, (mmol/L) & $5.81 \pm 0.54$ & $5.60 \pm 0.73$ & $5.63 \pm 0.73$ & $5.65 \pm 0.62$ & $5.60 \pm 0.70$ \\
\hline LDL-C (mmol/L) & $3.83 \pm 0.50$ & $3.61 \pm 0.61$ & $3.60 \pm 0.55$ & $3.59 \pm 0.56$ & $3.62 \pm 0.56$ \\
\hline ApoA-1 (gr/L) & $1.44 \pm 0.18$ & $1.41 \pm 0.15$ & $1.41 \pm 0.17$ & $1.41 \pm 0.17$ & $1.40 \pm 0.20$ \\
\hline ApoB (gr/L) & $1.04 \pm 0.12$ & $0.99 \pm 0.19$ & $1.01 \pm 0.16$ & $1.00 \pm 0.16$ & $1.00 \pm 0.13$ \\
\hline ApoE (gr/L) & $0.038 \pm 0.009$ & $0.039 \pm 0.010$ & $0.037 \pm 0.010$ & $0.040 \pm 0.015$ & $0.037 \pm 0.009$ \\
\hline PreBeta-1 (mg/dL) & $13.9 \pm 3.4$ & $\mathrm{n} / \mathrm{a}$ & $14.7 \pm 3.8$ & $13.8 \pm 4.1$ & $13.3 \pm 3.4$ \\
\hline PreBeta-2 (mg/dL) & $4.1 \pm 1.1$ & $\mathrm{n} / \mathrm{a}$ & $4.1 \pm 1.3$ & $4.3 \pm 1.3$ & $4.1 \pm 1.2$ \\
\hline Total CEC & $12.7 \pm 2.3$ & $12.5 \pm 2.4$ & $10.8 \pm 1.8$ & $12.5 \pm 2.6$ & $12.3 \pm 2.2$ \\
\hline ABCA1-specific CEC & $6.9 \pm 1.8$ & $6.6 \pm 1.9$ & $5.3 \pm 1.5$ & $6.7 \pm 1.9$ & $6.5 \pm 1.8$ \\
\hline Non-ABCA1-specific CEC & $5.8 \pm 1.0$ & $5.9 \pm 0.9$ & $5.5 \pm 0.7$ & $5.7 \pm 1.0$ & $5.8 \pm 0.9$ \\
\hline
\end{tabular}

BMI, body mass index; CEC, cholesterol efflux capacity; HDL-C, high-density lipoprotein cholesterol; LDL-C, low-density lipoprotein cholesterol; $n / a$, not available.

Data are presented as mean \pm standard deviation. 
Table S2 HDL- and CETP-related parameters and cholesterol efflux capacity at week 12 per group

\begin{tabular}{lccccc}
\hline Characteristics & $\begin{array}{l}\text { Placebo } \\
(\mathrm{n}=37)\end{array}$ & $\begin{array}{l}1 \mathrm{mg} \mathrm{TA}-8995 \\
(\mathrm{n}=37)\end{array}$ & $\begin{array}{l}5 \mathrm{mg} \mathrm{TA}-8995 \\
(\mathrm{n}=39)\end{array}$ & $\begin{array}{l}10 \mathrm{mg} \mathrm{TA}-8995 \\
(\mathrm{n}=35)\end{array}$ & $\begin{array}{c}10 \mathrm{mg} \text { TA-8995 }+10 \mathrm{mg} \\
\text { rosuvastatin }(\mathrm{n}=39)\end{array}$ \\
\hline Total cholesterol, (mmol/L) & $5.78 \pm 0.64$ & $5.49 \pm 0.89$ & $5.74 \pm 0.80$ & $6.10 \pm 0.72$ & $5.15 \pm 0.84$ \\
LDL-C (mmol/L) & $3.82 \pm 0.52$ & $2.62 \pm 0.60$ & $1.86 \pm 0.45$ & $1.87 \pm 0.53$ & $1.32 \pm 0.56$ \\
HDL-C (mmol/L) & $1.36 \pm 0.26$ & $2.36 \pm 0.54$ & $3.29 \pm 0.53$ & $3.61 \pm 0.61$ & $3.36 \pm 0.65$ \\
Triglycerides (mmol/L) & $1.27(0.88-1.47)$ & $1.03(0.89-1.23)$ & $1.14(0.98-1.49)$ & $1.18(0.92-1.49)$ & $0.95(0.81-1.23)$ \\
ApoA-1 (gr/L) & $1.46 \pm 0.21$ & $1.86 \pm 0.29$ & $2.22 \pm 0.28$ & $2.31 \pm 0.23$ & $2.16 \pm 0.26$ \\
ApoB (gr/L) & $1.02 \pm 0.14$ & $0.78 \pm 0.14$ & $0.66 \pm 0.10$ & $0.65 \pm 0.12$ & $0.54 \pm 0.12$ \\
ApoE (gr/L) & $0.038 \pm 0.10$ & $0.046 \pm 0.18$ & $0.049 \pm 0.016$ & $0.061 \pm 0.025$ & $0.055 \pm 0.017$ \\
PreBeta-1 (mg/dL) & $14.0 \pm 3.7$ & $\mathrm{n} / \mathrm{a}$ & $19.0 \pm 6.1$ & $18.0 \pm 8.2$ & $16.2 \pm 5.3$ \\
PreBeta-2 (mg/dL) & $4.3 \pm 1.1$ & $\mathrm{n} / \mathrm{a}$ & $6.3 \pm 1.9$ & $7.4 \pm 2.0$ & $6.7 \pm 2.3$ \\
Alpha-4 (mg/dL) & $16.4 \pm 3.2$ & $\mathrm{n} / \mathrm{a}$ & $16.3 \pm 3.6$ & $16.5 \pm 4.8$ & $15.8 \pm 3.3$ \\
Alpha-3 (mg/dL) & $27.1 \pm 5.6$ & $\mathrm{n} / \mathrm{a}$ & $19.7 \pm 3.6$ & $20.6 \pm 4.2$ & $19.7 \pm 3.2$ \\
Alpha-2 (mg/dL) & $55.6 \pm 11.9$ & $\mathrm{n} / \mathrm{a}$ & $55.4 \pm 9.4$ & $57.2 \pm 9.7$ & $53.6 \pm 8.3$ \\
Alpha-1 (mg/dL) & $25.5 \pm 8.6$ & $\mathrm{n} / \mathrm{a}$ & $97.5 \pm 20.1$ & $110.9 \pm 24.5$ & $104.8 \pm 27.9$ \\
Total CEC & $13.0 \pm 2.8$ & $15.2 \pm 3.2$ & $15.6 \pm 2.3$ & $18.2 \pm 4.1$ & $17.0 \pm 3.4$ \\
ABCA1-specific CEC & $7.0 \pm 2.2$ & $7.1 \pm 2.1$ & $6.6 \pm 1.7$ & $8.4 \pm 2.9$ & $7.3 \pm 2.3$ \\
Non-ABCA1-specific CEC & $6.0 \pm 1.0$ & $8.0 \pm 1.4$ & $9.0 \pm 1.1$ & $9.8 \pm 1.7$ & $9.7 \pm 1.6$ \\
\hline
\end{tabular}

CEC, cholesterol efflux capacity; HDL-C, high-density lipoprotein cholesterol; LDL-C, low-density lipoprotein cholesterol; $n / a$, not available. Data are presented as mean \pm SD. 
Table S3 Percentage change from baseline for HDL-subparticles and Cholesterol Efflux Capacity at week 12 per group

\begin{tabular}{lclccc}
\hline & $\begin{array}{l}\text { Placebo } \\
\text { Characteristics }\end{array}$ & $\begin{array}{l}1 \mathrm{mg} \mathrm{TA-8995} \\
(\mathrm{n}=37)\end{array}$ & $\begin{array}{l}5 \mathrm{mg} \mathrm{TA-8995} \\
(\mathrm{n}=37)\end{array}$ & $\begin{array}{l}10 \mathrm{mg} \text { TA-8995 } \\
(\mathrm{n}=35)\end{array}$ & $\begin{array}{c}10 \mathrm{mg} \text { TA-8995 }+10 \mathrm{mg} \\
\text { rosuvastatin }(\mathrm{n}=39)\end{array}$ \\
\hline Total cholesterol (mmol/L) & $-0.4 \pm 9.1$ & $-1.4 \pm 13.5$ & $2.3 \pm 10.9$ & $8.8 \pm 15.1^{* *}$ & $-7.8 \pm 11.0$ \\
LDL-C (mmol/L) & $0.2 \pm 11.7$ & $-27.0 \pm 13.1^{* * *}$ & $-48.7 \pm 9.1^{* * *}$ & $-46.4 \pm 16.8^{* * *}$ & $-63.7 \pm 15.7^{* * *}$ \\
HDL-C (mmol/L) & $1.8 \pm 11.0$ & $76 \pm 26.4^{* * *}$ & $160.9 \pm 42.3^{* * *}$ & $180.6 \pm 42.1^{* * *}$ & $156.5 \pm 52.0^{* * *}$ \\
Triglycerides (mmol/L) & $-2.6 \pm 28.9$ & $-16.7 \pm 21$ & $-11.4 \pm 28.5$ & $-7.7 \pm 42.3$ & $-17.8 \pm 28.9$ \\
ApoA-1 (gr/L) & $1.5 \pm 10.7$ & $31.9 \pm 15.8^{* * *}$ & $58.3 \pm 18.2^{* * *}$ & $65.1 \pm 18.6^{* * *}$ & $55.8 \pm 22.4^{* * *}$ \\
ApoB (gr/L) & $-0.5 \pm 13.2$ & $-19.2 \pm 14.1^{* * *}$ & $-33.8 \pm 11.1^{* * *}$ & $-33.6 \pm 15.3^{* * *}$ & $-45.3 \pm 12.3^{* * *}$ \\
PreBeta-1 (\%) & $1.9 \pm 6.9$ & $\mathrm{n} / \mathrm{a}$ & $34.6 \pm 6.7^{* *}$ & $36.9 \pm 7.1^{* *}$ & $21.5 \pm 6.6$ \\
PreBeta-2 (\%) & $7.0 \pm 5.0$ & $\mathrm{n} / \mathrm{a}$ & $55.8 \pm 4.9^{* * *}$ & $76.9+5.2^{* * *}$ & $63.3 \pm 4.8^{* * *}$ \\
Alpha-1 (\%) & $7.1 \pm 17.9$ & $\mathrm{n} / \mathrm{a}$ & $337.6 \pm 17.5^{* * *}$ & $364.6 \pm 18.4^{* * *}$ & $352.1 \pm 17.2^{* * *}$ \\
Alpha-2 (\%) & $2.2 \pm 3.2$ & $\mathrm{n} / \mathrm{a}$ & $7.0 \pm 3.1$ & $5.8 \pm 3.3$ & $3.8 \pm 3.1$ \\
Alpha-3 (\%) & $8.4 \pm 2.9$ & $\mathrm{n} / \mathrm{a}$ & $-18.9 \pm 2.8^{* * *}$ & $-16.6 \pm 3.0^{* * *}$ & $-17.2 \pm 2.8^{* * *}$ \\
Alpha-4 (\%) & $1.3 \pm 3.7$ & $\mathrm{n} / \mathrm{a}$ & $4.5 \pm 3.7$ & $2.7 \pm 3.8$ & $-1.7 \pm 3.6$ \\
Total CEC (\%) & $-6.7 \pm 2.9$ & $16.8 \pm 2.9^{* * *}$ & $33.2 \pm 2.8^{* * *}$ & $38.3 \pm 3.0^{* * *}$ & $31.1 \pm 2.8^{* * *}$ \\
ABCA1 CEC (\%) & $3.9 \pm 5.0$ & $13.5 \pm 5.0$ & $24.8 \pm 5.1^{*}$ & $28.3 \pm 5.1^{* *}$ & $15.6 \pm 4.8$ \\
Non-ABCA1 CEC (\%) & $4.3 \pm 3.0$ & $37.8 \pm 3.0^{* * *}$ & $62.4 \pm 3.0^{* * *}$ & $72.2 \pm 3.1^{* * *}$ & $67.4 \pm 2.9^{* * *}$ \\
\hline
\end{tabular}

CEC, cholesterol efflux capacity; HDL-C, high-density lipoprotein cholesterol; LDL-C, low-density lipoprotein cholesterol; n/a, not available.

Data are presented as least-squares means. For $1 \mathrm{mg}$ of TA-8995, no $2 \mathrm{D}$ gel electrophoresis data are available. ${ }^{\star} P<.05 ;{ }^{*} P<.01 ;{ }^{* *} P<.001$ compared to placebo. 